\title{
Ultraproducts and higher order models
}

\section{Wilfred Gordon Malcolm}

The programme of work for this thesis began with the somewhat general intention of paralleling in the context of higher order models the ultraproduct construction and its consequences as developed in the literature for first order models. Something of this was, of course, already available in the ultraproduct construction of W.A.J. Luxemburg used in Non Standard Analysis.

It may have been considered that such a general intention was not likely to yield anything of significance over and above what was already available from viewing the higher order situation as a 'many sorted' first order one and interpreting the first order theory accordingly. In the event, however, this seems not to be so. In particular the substructure concepts developed in Chapter II of the main thesis together with the various embedding theorems and their applications are not immediately available from the first order theory and seem to be of sufficient worth to warrant developing the higher order theory in its own terms. This, anyway, is the basic justificaition for the approach and content of the thesis.

In Chapter I, apart from reviewing the standard concepts for higher order models and the related ultraproduct construction, some attention is given to the concepts of normality and fullness and the manner in which these find expression in the ultraproduct construction. Model theoretic results paralleling those of a first order theory and ultraproducts are established.

In the first section of Chapter II a substructure concept for higher order models is defined and some of its properties established. In the second section various theorems involving the embedding of a structure into

Received 6 December 1972. Thesis submitted to the Victoria University of Wellington, August 1972. Degree approved, December 1972. Supervisors: Dr M.J. Cresswell, Professor G.E. Hughes, Professor C.J. Seelye. 
an ultraproduct of a local family of its substructures are proved. Brief mention is given to the presence of 'inverse limits' in this embedding context.

In Chapter III various algebraic applications are given of the concepts and theorems of Chapter II. In Section 1, Stone's Representation Theorem for boolean algebras is expressed as an example of an ultraproductembedding. In Section 2 the presence of a higher order ultraproduct construction in the mechanism of Sylow p-subgroups of locally normal groups is exposed and analysed. Perhaps the most significant and fruitful applications are those in Sections 3 and 4 concerned with the theory of local properties in universal algebras.

Two appendices are included. The first discusses modifications to a compactness result for a generalised first order language established by Shafaat [2]. The second develops some consequences to simple variations in the first order ultraproduct construction.

Much of the material in the thesis has already been accepted for publication in the papers as listed below [1].

\section{References}

[1] W.G. Malcolm, "Variations in definition of ultraproducts of a family of first order relational structures", Notre Dome J. Formal Logic 13 (1972), 394-398.

W.G. Malcolm, "On a compactness theorem of A. Shafaat", J. London Math. Soc. (2) 5 (1972), 719-725.

W.G. Malcolm, "Some results and algebraic applications in the theory of higher order ultraproducts", Notre Dame J. Formal Logic (to appear).

W.G. Malcolm, "Application of higher order ultraproducts to the theory of local properties in universal algebras", Proc. London Math. Soc. (to appear).

[2] A. Shafaat, "Principle of localisation for a more general type of Ianguages", Proc. London Math. Soc. (3) 17 (1967), 629-643. 